\title{
Management of labor and delivery by spinal and epidural analgesia in a woman with systemic mastocytosis: a case report
}

\author{
Chiharu Kawagoe $^{1}$, Chiyo Ootaki $^{1}$, Yuki Kinishi ${ }^{1}$, Chie Matsuda ${ }^{1}$, and Reiko Uokawa. ${ }^{2}$ \\ ${ }^{1}$ Osaka University School of Medicine Graduate School of Medicine \\ ${ }^{2}$ Osaka University Faculty of Medicine Graduate School of Medicine
}

February 19, 2021

\begin{abstract}
Systemic mastocytosis is a life-threatening disease in which mast cell mediator release can lead to general symptoms. The most common triggers are stress and pain during labor and delivery. We report the management of labor and delivery in a case with severe systemic mastocytosis by epidural analgesia.
\end{abstract}

\section{INTRODUCTION}

Mastocytosis is a rare disease caused by the abnormal proliferation of mast cells. The prevalence of mastocytosis is estimated to be 13 per 100000 in all age categories [1,2]. Systemic mastocytosis is a genetic disorder and is most commonly caused by a point mutation in the gene for tyrosine kinase receptor Kit D816 V. The symptoms of mastocytosis are quite diverse as mast cells accumulate in various organs, especially the bone marrow, skin, liver, spleen, and lymph nodes. Histamine, heparin, and tryptase are the most recognized preformed mediators that are released from mast cells. Newly formed mediators at the time of the reaction include prostaglandins, leukotrienes, chemokines and cytokines [3]. Mast cell mediator release can lead to general symptoms such as abdominal pain, urticaria, pruritus, syncope, and vascular collapse, including death in severe cases. Common triggers are insect stings and drugs, including nonsteroidal anti-inflammatory drugs (NSAIDs), anesthetics, antibiotics, food, and mechanical stimulation [2]. Stress and pain during labor and delivery can activate the disease $[3,4]$. Here, we report the case of a pregnant woman with systemic mastocytosis who received epidural analgesia to avoid labor pain.

\section{CASE PRESENTATION}

A 38-year-old woman at 39 weeks 6 days gestation (G1P0, height $159 \mathrm{~cm}$, weight $66 \mathrm{~kg}, 52 \mathrm{~kg}$ prepregnancy) presented to our hospital with labor pains. She had a long history of mastocytosis, with the development of pale brown chromatosis and hives from the back to the buttocks starting at 26 years old. Her symptoms worsened at 29 years old, and a wheal appeared while she was wearing and removing clothes. At the age of 32 , the skin eruption worsened, and she was diagnosed with urticaria pigmentosa by a skin biopsy. She developed anaphylaxis twice with generalized skin rash and dyspnea in response to ibuprofen and diclofenac, and she was transferred to the emergency department and treated with intravenous betamethasone both times. Six months later, a skin biopsy and bone marrow biopsy performed at another hospital revealed systemic mastocytosis. She experienced convulsions after the use of an over-the-counter cold remedy and developed systemic erythema and dyspnea after the use of loxoprofen. Before fertility treatment, an allergy test was performed to identify the drugs that could be used during delivery, but no tests were performed for the drugs that can be used for labor analgesia. She became pregnant by in vitro fertilization, and her pregnancy progressed smoothly. She was on famotidine and ebastine for systemic mastocytosis before 
pregnancy and took additional fexofenadine hydrochloride when her condition worsened. She regularly took antihistamines and fewer oral steroids after the pregnancy.

During her pregnancy, the obstetricians, dermatologists, immunologists, hematologists, and anesthesiologists discussed various delivery plans and opted for epidural analgesia to avoid mast cell degranulation and anaphylaxis. The obstetrician planned a vaginal delivery without induction, based on evidence; systemic mastocytosis itself is not an indication for cesarean section. The patient received a prenatal anesthetic evaluation and was given an explanation of the anesthesia plan: epidural analgesia would be placed to avoid stress, which could trigger mast cell degranulation.

At 39 weeks 6 days of gestation, she complained of strong pain at the time she was admitted to the hospital. At admission, a rash and general cold sweat were found on her anterior chest wall and back. We suspected labor pain and psychological stress as the triggers for those symptoms and administered antihistamines and performed a combined spinal-epidural analgesia (CSEA). Before anesthesia, there were no abnormal laboratory data. The CSEA was placed at the L3-L4 level, and $1.5 \mathrm{mg} 0.5 \%$ isobaric bupivacaine with fentanyl $15 \mu \mathrm{g}$ was injected into the subarachnoid space, then a catheter was introduced into the epidural space at a cervical dilation of $4 \mathrm{~cm}$ and a pain NRS (Numerical Rating Scale) of 10. After placing the CSEA, her NRS became 0, and her sensory analgesia level was confirmed on both sides from Th5 to S5. Two hours after placing the CSE, her labor analgesia was switched to a programmed intermittent epidural bolus (PIEB) with $0.1 \%$ ropivacaine, and fentanyl $2 \mu \mathrm{g} / \mathrm{mL}$ was set at $8 \mathrm{~mL}$ with a 60 -minute interval. Her analgesia level was kept at NRS 3-4, and her anesthesia level was kept at Th 8.9-S3 area until delivery. When the cervix was fully dilated, a rash and pruritus were noted on her abdomen, but sufficient pain relief was obtained with an NSR score of 3. H1 and H2 antihistamines were administered, the pruritus was suppressed, and vaginal delivery was performed as scheduled. The female infant weighed $3246 \mathrm{~g}$ and had Apgar scores of 7 and 9 at 1 and 5 minutes, respectively. The patient and the infant had an uncomplicated postpartum course and were discharged on postpartum day 5 .

\section{DISCUSSION}

We present a case of pregnancy complicated by the need for the management of systemic mastocytosis with several prior anaphylaxis episodes. We applied epidural analgesia to prevent exacerbation of the disease and anaphylaxis during labor and delivery. Multiple stimuli can provoke mast cell degranulation. The triggers include physical, chemical and biologic agents [3,5]. Stress is also a well-known trigger for mediator release in systemic mastocytosis [3,4]. Pregnancy complications can be associated with increased levels of maternal stress, and labor is clearly a time of increased maternal pain and stress [3]. Based on the hypothesis that good pain management may contribute to reducing stress and reducing the risk of exacerbation of systemic mastocytosis, there are several prior reports of epidural anesthesia for pain relief during labor in pregnant patients with systemic mastocytosis. [6,7]. We planned early epidural placement to avoid stress and labor pain, which can trigger systemic mastocytosis.

However, anaphylactoid reactions may also be triggered by drugs administered during delivery, especially anesthesia. Testing for drug hypersensitivity is generally recommended only in patients with a history of drug allergy [8]. This patient had a history of drug allergy. However, in this case, labor analgesics and general anesthetics were not tested during pregnancy while planning the labor analgesia because the allergy/immunology physician did not recommend it as the testing could trigger mast cell degranulation. Therefore, we avoided the use of anesthetics such as morphine, NSADs, meperdine, and ester local anesthetics, which could trigger mast cell degranulation during labor analgesia [9].

This patient had an NRS score of 10 at the time she was admitted to the hospital and complained of very strong pain. Although it should have been introduced by the straight epidural method assuming the possibility of fetal distress and a possible emergency cesarean section, the CSE method was selected by the anesthesiologist in charge because CSE was the most commonly used method. As our facility uses PIEB as a standard, the level of anesthesia and her vitality were checked every hour to avoid unexpected hypotension or high epidural levels. 
Mastocytosis is subdivided into two categories as proposed by the WHO: cutaneous and systemic mastocytosis $[10,11]$. Cutaneous mastocytosis is the most common form of mastocytosis, which usually occurs in childhood and appears as hyperplasia of the mast cells of the skin [12]. Systemic mastocytosis is a very rare disease in which myeloproliferative tumors derived from mast cells form multifocal lesions and often infiltrate the skin, lymph nodes, digestive organs, liver, and spleen. The diagnosis of systemic mastocytosis is based on the presence of one major criterion and one minor criterion or three minor criteria (Table 1). Symptoms of systemic mastocytosis include abdominal pain, diarrhea, nausea, vomiting, skin pruritus and flushing, anaphylactoid reactions, anemia, and pathological fractures. Our patient was first diagnosed with urticaria pigmentosa, but later, she was diagnosed with systemic mastocytosis by observing the proliferation of mast cells in skin and bone marrow biopsies.

Approximately $22 \%$ to $33 \%$ of pregnant women with systemic mastocytosis experience worsening of systemic symptoms due to increased physical and psychological stress during pregnancy $[6,9,13]$. Theoretically, hormonal changes associated with pregnancy and puerperium may promote anaphylactic reactions. There are reports of anaphylactic episodes during pregnancy [14]. Exacerbations of systemic mastocytosis during pregnancy can cause life-threatening maternal and fetal complications. There have been several reports of preterm labor and deliveries [7,9]. There is also a case report of a fetal death due to an acute exacerbation of systemic mastocytosis [3]. This fetal demise that occurred at 31 weeks of gestation was caused by an exacerbation of systemic mastocytosis, which resulted in severe hypotension and a critical decrease in uterine blood flow.

In our case, there were no significant changes during pregnancy. The anesthesiologist should prepare a plan for analgesia and anesthesia for delivery to minimize the risk. Good pain control helps reduce overall anxiety and reduces the risk of exacerbated mastocytosis during labor. Our patient presented with minor skin symptoms during labor pain, but it improved with antihistamines and immediate pain relief from epidural analgesia without any serious complications.

\section{CONCLUSION}

For the effective management of pregnant women with mastocytosis, triggers of mast cell degranulation should be avoided. Early epidural analgesia is effective for relieving stress and pain during labor, and it is necessary to avoid drugs that induce histamine release and to use drugs that suppress it. The team involved in the delivery, including anesthesiologists, obstetricians, and midwives, should discuss this with patients with systemic mastocytosis from pregnancy to after delivery to prevent an acute exacerbation of their symptoms.

\section{CONFLICTS OF INTEREST}

The authors declare that they have no competing interests.

\section{AUTHOR CONTRIBUTIONS}

CK drafted the manuscript. CK, CM, YK and RU carried out the anesthesia for the entire course. CO supervised the drafting. All authors read and approved the final version of the manuscript.

\section{CONSENT TO PARTICIPATE}

Informed consent for scientific publication was obtained from the patient.

\section{ACKNOWLEDGMENTS}

The authors would like to extend their gratitude to the patient, her family members and Osaka University obstetricians, dermatologists, immunologists, and hematologists.

\section{REFERENCES}

1. van Doormaal JJ, Arends S, Brunekreeft KL, et al. Prevalence of indolent systemic mastocytosis in a Dutch region. Allergy Clin Immunol. 2013;131(5):1429-1431. 
2. Ciach K, Niedoszytko M, Abacjew-Chmylko A, et al. Pregnancy and Delivery in Patients with Mastocytosis Treated at the Polish Center of the European Competence Network on Mastocytosis (ECNM). Plos One. 2016;21;11(1) doi:10.1371/journal.pone.0146924.

3. Kendra D. Watson, Katherine W. Arendt, MD, et al. Volcheck, MD. Systemic Mastocytosis Complicating Pregnancy. Obstet Gynecol. 2012;119(2):486-489.

4. Theoharides TC. The impact of psychological stress on mast cells. Ann Allergy Asthma Immunol. 2020;125(4):388-392.doi: 10.1016/j.anai.2020.07.007.

5. Brockow K, Jofer C, Behrendt H, Ring J. Anaphylaxis in patients with mastocytosis: a study on history, clinical features and risk factors in 120 patients. Allergy. 2008;63(2):226-232.

6. Villeneuve V, Kaufman I, Weeks S, Deschamps A. Anesthetic management of a labouring parturient with urticaria pigmentosa. Can J Anaesth. 2006;53(4):380-384.

7. Matito A, Álvarez-TwoseI, Morgado JM, et al. Clinical impact of pregnancy in mastocytosis: a study of the Spanish Network on Mastocytosis (REMA) in 45 cases. Int Arch Allergy Immunol. 2011;156(1):104-111.

8. Metcalfe DD, Akin C. Mastocytosis: molecular mechanisms and clinical disease heterogeneity. Leuk Res. 2001; 25:577-82.

9. Ulbrich F, Engelstädter N, et al. Anaesthetic management of emergency caesarean section in a parturient with systemic mastocytosis. Int J Obstet Anesth. 2013;22(3):243-246.

10. Madendag IC, Madendag Y, Tarhan I, et al. Mastocytosis in pregnancy. Taiwan J Obstet Gynecol. 2010;49(2):192-196.

11. Valent P, Akin C, Escribano L, et al. Standards and standardization in mastocytosis: consensus statements on diagnostics, treatment recommendations and response criteria. Eur J Clin Invest. 2007;37(6):435-453.

12. Akin C, Metcalfe DD. Systemic mastocytosis. Annu Rev Med. 2004;55:419-432.

13. Dawn Lei, Cem Akin, Anna Kovalszki. Management of Mastocytosis in Pregnancy: A Review. J Allergy Clin Immunol Pract. 2017;5(5):1217-1223.

14. Kendra D. Watson, Katherine W. Arendt, MD, et al. Volcheck, MD. Systemic Mastocytosis Complicating Pregnancy. Obstet Gynecol. 2012;119(2):486-489. 